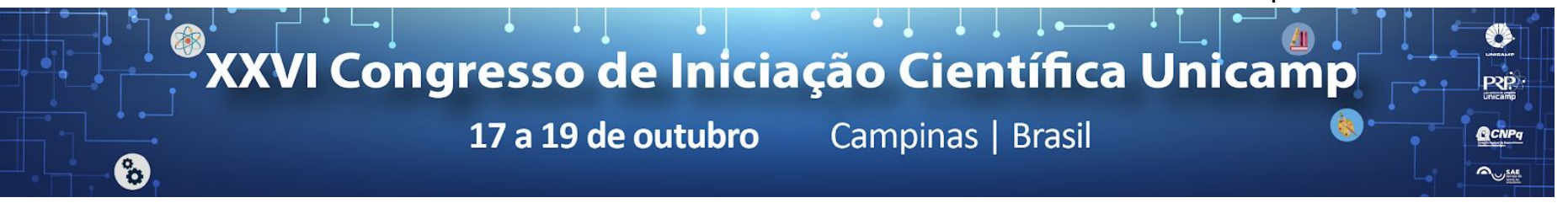

\title{
The psychology of representation and the information design applied into the architectural diagram analysis.
}

\section{Raissa A. Rodrigues*, Daniel de Carvalho Moreira}

\begin{abstract}
Sketches are contiguous tools for architects during the design process, especially in the conception phase whereas they are able to convey agile a mental idea. Taking into account that to concept is, for many architects, a fundamental part of the process, the research focus is concerning the usage, often natural, of diagrammatic resources. These media are fundamental to express and to order the complex amount of information inherent in the architecture project, in its various phases, such as in sketches, for the purpose of becoming communication increasingly more synthetic. Toward sketches analysis, a chart was design, based on psychology of representation studies and information design. Both areas analyze technical, communicative and cognitive aspects of drawing, considering the intrinsic relation between the representation, the conception and the perception of the project. The result suggests that each sketch has a specific purpose and a concept to be clarified, which is directly influenced by the type of representation, adopted by a certain perception of the architect.
\end{abstract}

\section{Key words:}

Sketch, architectural diagram, graphic analysis.

\section{Introduction}

Architectural diagrams are inherent concepts when understood as graphical information representations. However, Architectural diagrams interpretations have been conducted comprehensively, through visual models and graphical analysis, neglecting the exploration of design and organizational strategies under the aspect of representation in architecture. A different approach is proposed, as a diagrammatic resource, that aims to clarify the purpose by confronting the technical, realistic and abstract perspectives of an idea. In sketches, the space is reduced to information and the information into interpretation, in order to represent synthetically the mental idea. From this point, sketches can be seen as architectural diagram holders.

Each sketch is different from the other, both in technical and communication aspects, since the type of drawing to be carried out is dependent on its function and the architect strategies for representing it by processes of emphasis and exclusion of what is significant. Those who draw, therefore, have possibility to choose which values or interpretations to assign among the innumerable possibilities.

\section{Results and Discussion}

The concepts of psychology of representation were studied to justify the selection of information design parameters for developing an analysis tool of diagrammatic resources in sketches. Based on technical, communicative and cognitive aspects, the research associate the perception to representation and conception in the design process, emphasizing the interdependence between these three fields and the idea that any reality graphic representation is always an particular and precise data interpretation of the same reality.

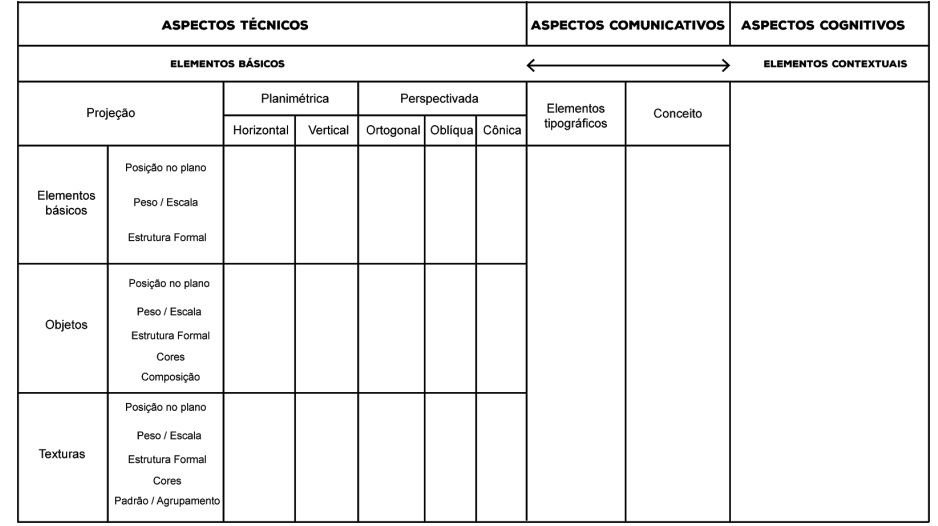

Chart 1. Sketches analysis structure. Source: Raissa A. Rodrigues.

Thus, the variation in types of representation influences the perspective of observing, conceiving and understanding the project, and the influence of perception shapes the primary elements representation.

\section{Conclusions}

It is expected to contribute with a system that not only uses the graphical analysis, but also it tries to gather and confront the several dimensions inherent in sketches, in order to find new means of thinking sketches and demonstrating its great potential to convey information and clarify concepts.

\section{Acknowledgement}

The author Raissa A. Rodrigues thanks her advisor Daniel de Carvalho Moreira; Ana Paula Rocha, who idealized the theme of the research; and her own beloved family, that vibrates by each step and each conquest. 